\title{
Article \\ External Validation and Extension of a Clinical Score for the Discrimination of Type 2 Myocardial Infarction
}

\author{
Thomas Nestelberger $1,2,3,+\left(\mathbb{D}\right.$, Pedro Lopez-Ayala $1,2,+\mathbb{D}$, Jasper Boeddinghaus ${ }^{1,2,+}$, Ivo Strebel ${ }^{1,2}$, \\ Maria Rubini Gimenez 1,2,4 , Iris Huber 1,2, Karin Wildi 1,2,5, Desiree Wussler 1,2, Luca Koechlin 1,2,6 $\mathbb{D}$, \\ Alexandra Prepoudis ${ }^{1,2} \mathbb{D}_{\text {, Danielle M. Gualandro }}{ }^{1,2}$, Christian Puelacher ${ }^{1,2} \mathbb{D}$, Noemi Glarner ${ }^{1,2} \mathbb{D}$, Philip Haaf 1 , \\ Simon Frey ${ }^{1}$ (D), Adam Bakula ${ }^{1}$, Rupprecht Wick ${ }^{1}$, Òscar Miró ${ }^{2,7}$, F. Javier Martin-Sanchez ${ }^{2,8}$, \\ Damian Kawecki ${ }^{2,9}$ D, Dagmar Keller ${ }^{10}$, Raphael Twerenbold ${ }^{1,2}$ and Christian Mueller 1,2,*,
}

\section{check for} updates

Citation: Nestelberger, T.; Lopez-Ayala, P.; Boeddinghaus, J.; Strebel, I.; Rubini Gimenez, M.; Huber, I.; Wildi, K.; Wussler, D.; Koechlin, L.; Prepoudis, A.; et al. External Validation and Extension of a Clinical Score for the Discrimination of Type 2 Myocardial Infarction. J. Clin. Med. 2021, 10, 1264. https:// doi.org/10.3390/jcm10061264

Academic Editor: Nicola Cosentino

Received: 3 March 2021

Accepted: 14 March 2021

Published: 18 March 2021

Publisher's Note: MDPI stays neutral with regard to jurisdictional claims in published maps and institutional affiliations.

Copyright: (c) 2021 by the authors. Licensee MDPI, Basel, Switzerland. This article is an open access article distributed under the terms and conditions of the Creative Commons Attribution (CC BY) license (https:// creativecommons.org/licenses/by/ $4.0 /$ )
1 Cardiovascular Research Institute Basel (CRIB) and Department of Cardiology, University Hospital Basel, University of Basel, 4031 Basel, Switzerland; thomas.nestelberger@usb.ch (T.N.); pedro.lopezayala@usb.ch (P.L.-A.); jasper.boeddinghaus@usb.ch (J.B.); ivo.strebel@usb.ch (I.S.); maria.rubini@usb.ch (M.R.G.); iris.huber@usb.ch (I.H.); wildik@hotmail.com (K.W.); desireenadine.wussler@usb.ch (D.W.); luca.koechlin@usb.ch (L.K.); alexandra.prepoudis@usb.ch (A.P.); danielle.gualandro@usb.ch (D.M.G.); christian.puelacher@usb.ch (C.P.); noemi.glarner@usb.ch (N.G.); philip.haaf@usb.ch (P.H.); simon.frey@usb.ch (S.F.); adam.bakula@pm.me (A.B.); Rupprecht.wick@usb.ch (R.W.); raphael.twerenbold@usb.ch (R.T.)

2 GREAT Network, 00191 Rome, Italy; omiro@clinic.cat (Ò.M.); fjjms@hotmail.com (F.J.M.-S.); d.kawecki@interia.pl (D.K.)

3 Division of Cardiology, Vancouver General Hospital, University of British Columbia, Vancouver, BC V5Z 1M9, Canada

4 Department of Internal Medicine/Cardiology, Heart Center Leipzig at University Leipzig, 04109 Leipzig, Germany

5 Critical Care Research Institute, the Prince Charles Hospital, Brisbane and University of Queensland, 4072 Brisbane, Australia

6 Department of Cardiac Surgery, University Hospital Basel, 3010 Basel, Switzerland

7 Emergency Department, Hospital Clinic, 08036 Barcelona, Spain

8 Servicio de Urgencias, Hospital Clínico San Carlos, 28040 Madrid, Spain

9 2nd Department of Cardiology, School of Medicine with the Division of Dentistry in Zabrze, Medical University of Silesia, 40-055 Katowice, Poland

10 Emergency Department, University Hospital Zurich, 8006 Zurich, Switzerland; dagmar.keller@usb.ch

* Correspondence: Christian.mueller@usb.ch; Tel.: +41-61-328-65-49; Fax: +41-61-265-53-53

$+\quad$ These three co-authors have made the equal contribution.

$\ddagger$ For the APACE Investigators.

Abstract: Background: The early non-invasive discrimination of Type 2 versus Type 1 Myocardial Infarction (T2MI, T1MI) is a major unmet clinical need. We aimed to externally validate a recently derived clinical score (Neumann) combing female sex, no radiating chest pain, and high-sensitivity cardiac troponin I (hs-cTnI) concentration $\leq 40.8 \mathrm{ng} / \mathrm{L}$. Methods: Patients presenting with acute chest discomfort to the emergency department were prospectively enrolled into an international multicenter diagnostic study. The final diagnoses of T2MI and T1MI were centrally adjudicated by two independent cardiologists using all information including cardiac imaging and serial measurements of hs-cTnT/I according to the fourth universal definition of MI. Model performance for T2MI diagnosis was assessed by formal tests and graphical means of discrimination and calibration. Results: Among 6684 enrolled patients, MI was the adjudicated final diagnosis in 1079 (19\%) patients, of which $242(22 \%)$ had T2MI. External validation of the Neumann Score showed a moderate discrimination (C-statistic 0.67 (95\%CI 0.64-0.71)). Model calibration showed underestimation of the predicted probabilities of having T2MI for low point scores. Model extension by adding the binary variable heart rate $>120 / \mathrm{min}$ significantly improved model performance (C-statistic 0.73 (95\% CI $0.70-0.76, p<0.001$ ) and had good calibration. Patients with the highest score values of 3 (Neumann Score, $9.9 \%$ ) and 5 (Extended Neumann Score, 3.3\%) had a 53\% and 91\% predicted probability of T2MI, respectively. Conclusion: The Neumann Score provided moderate discrimination and suboptimal calibration. Extending the Neumann Score by adding heart rate $>120 / \mathrm{min}$ improved the model's performance. 
Keywords: type 1 myocardial infarction; type 2 myocardial infarction; differentiation; external validation; risk scores

\section{Introduction}

Myocardial infarction (MI) remains the most common cause of death worldwide [1,2]. The clinical introduction of high-sensitivity cardiac troponin (hs-cTn) assays has enabled a more accurate diagnosis of MI [1,2]. Furthermore, it has facilitated the recognition that in a relevant proportion of patients with MIs, supply-demand mismatch due to impaired systemic hemodynamics including hypotension, hypertension, tachycardia, or hypoxemia - rather than coronary atherothrombosis (Type $1 \mathrm{MI}$ (T1MI))-is the underlying pathophysiology (Type 2 MI (T2MI)) [3-9]. As treatments differ substantially between T2MI and T1MI, [1,2] their early and accurate non-invasive differentiation is a major, yet largely unmet clinical need. When taken individually, clinical characteristics have limited diagnostic accuracy for the early non-invasive differentiation of T2MI versus T1MI. A recent single-center pilot study introduced a multivariable score (Neumann Score) that includes female sex, no radiating chest pain, and hs-cTnI $\leq 40.8 \mathrm{ng} / \mathrm{L}$ (Abbott Architect) for the differentiation between T2MI and T1MI in the emergency department (ED) [10].

We therefore aimed to, first, externally validate the Neumann Score for the discrimination of T2MI versus T1MI in a large international diagnostic multicenter study and, second, assess whether its performance can be improved by incorporating other routinely available clinical variables.

\section{Methods}

\subsection{Study Design and Population}

Advantageous Predictors of Acute Coronary Syndrome Evaluation (APACE) is an ongoing prospective multicenter international diagnostic study including 12 centers in 5 countries aiming to advance the early diagnosis of MI (ClinicalTrials.gov (accessed on 3 March 2021) registry, number NCT00470587) [5].

Adult patients presenting to the ED with symptoms suggestive of MI, such as acute chest discomfort or angina pectoris with a chest pain onset or maximum within $12 \mathrm{~h}$ prior to presentation, were recruited. For this analysis, patients were excluded if (A) they presented with ST-elevation myocardial infarction (STEMI), as T2MI rarely presents as STEMI, (B) the final diagnosis remained unclear even after final adjudication and had at least one elevated hs-cTn concentration, thereby possibly indicating MI, (C) patients presenting with chest pain onset/maximum $>12 \mathrm{~h}$, (D) terminal kidney failure requiring dialysis, (E) final adjudication was other than MI, as the Neumann Score is applied only for MI patients, and (F) a component of the Neumann Score was missing. (Figure 1).

The study was carried out according to the principles of the Declaration of Helsinki and approved by the local ethics committees. Written informed consent was obtained from all patients. The authors designed the study, gathered, and analyzed the data according to the Transparent Reporting of a multivariable prediction model for Individual Prognosis or Diagnosis (TRIPOD) statement (Supplementary Table S1) [11], vouched for the data and analysis, wrote the paper, and decided to publish. The assays were donated by the manufacturers, who had no role in the design of the study, data analysis, manuscript preparation, or decision to submit for publication.

\subsection{Routine Clinical Assessment}

All patients underwent clinical assessment that included standardized and detailed medical history, vital signs, physical examination, 12-lead electrocardiogram (ECG), continuous ECG rhythm monitoring, pulse oximetry, standard blood test, as well as non-invasive and invasive cardiac imaging as indicated. Vital signs and chest pain characteristics used for the score were assessed immediately after ED presentation. Cardiac troponin levels, 
including hs-cTn in some centers, were measured at presentation and serially thereafter if clinically indicated. Treatment of patients was left to the discretion of the attending physician. The estimated glomerular filtration rate (eGFR) was estimated using the chronic kidney disease epidemiology collaboration (chronic kidney disease-modification of diet in renal disease; CKD-MDRD) formula [12].

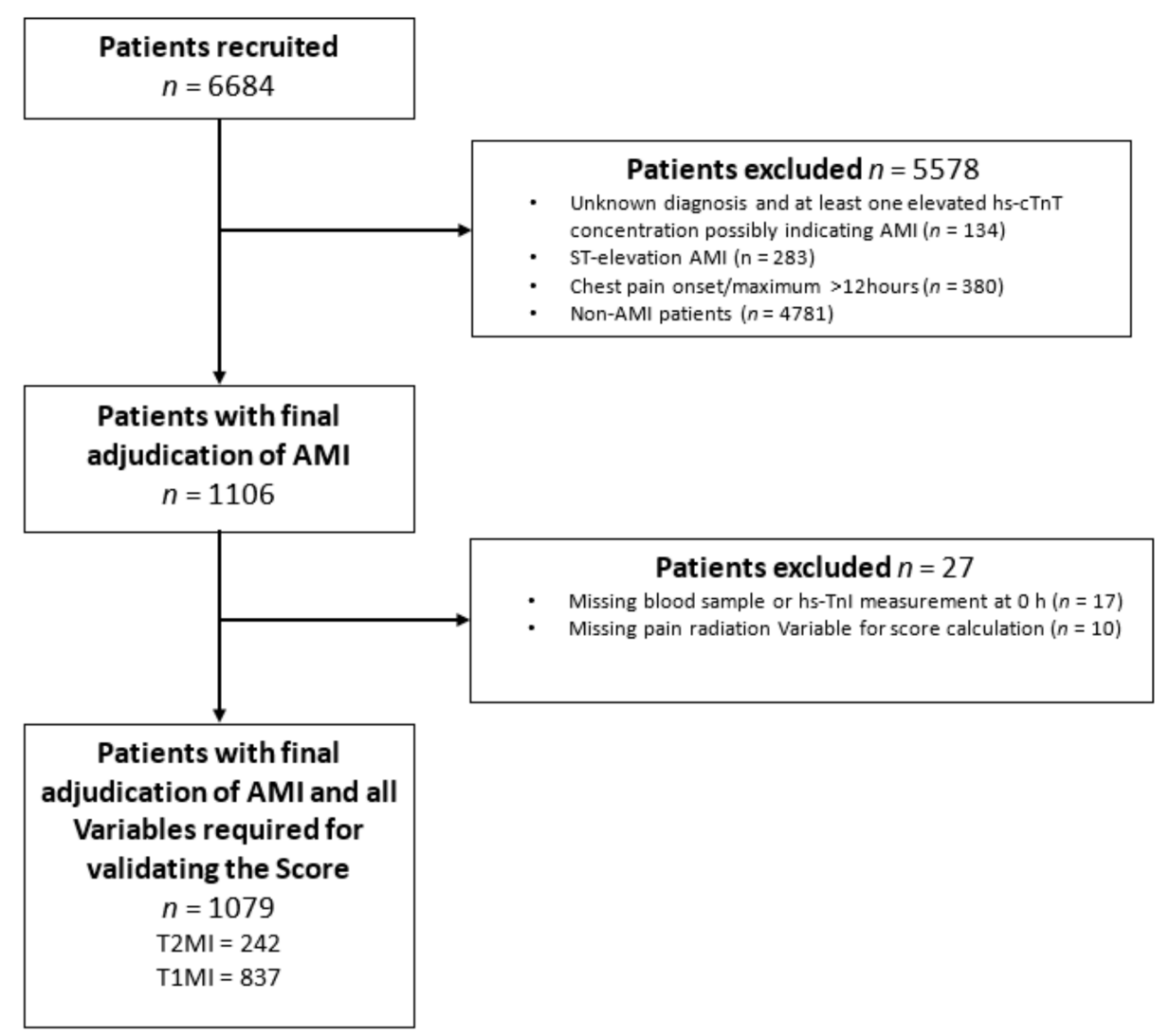

Figure 1. Patient flow. AMI, acute myocardial infarction, hs-cTnI, high-sensitivity cardiac Troponin I.

\subsection{Central Adjudication of T1MI and T2MI}

Two independent cardiologists reviewed all available medical records including patient history, physical examination, vital signs in the ambulance and in the ED, results of laboratory testing, radiologic testing, ECG, echocardiography, cardiac magnetic resonance imaging, lesion severity and morphology in coronary angiography pertaining to the patient. In situations of disagreement about the diagnosis, cases were reviewed and adjudicated in conjunction with a third cardiologist. Adjudication of the final diagnosis was performed centrally in a core laboratory and included two sets of serial (hs-)cTn measurements: serial (hs)-cTn measurements obtained as part of routine clinical care locally (different (hs)-cTn assays) and serial measurements of hs-cTnT from study blood draws performed centrally in the core laboratory in order to take advantage of the higher sensitivity and higher overall diagnostic accuracy offered by hs-cTnT [2].

T1MI and T2MI were defined according to the fourth universal definition of MI [2]. In addition to the evidence of myocardial necrosis in a clinical setting consistent with acute myocardial ischemia, T1MI was defined as spontaneous MI related to a primary atherothrombotic coronary event such as plaque erosion or rupture, intraluminal coronary thrombus, or distal microembolization. T2MI was defined as secondary to an oxygen supply-demand mismatch in the context of brady- or tachyarrhythmias, hypoxemia, hypotension, hypertension, severe anemia, or coronary artery spasm, coronary embolism, and non-atherosclerotic dissection. An underlying coronary artery disease was possible, but not required, for T2MI. To qualify for T2MI, the same dynamic changes in hs-cTn were 
required as for T1MI. As recommended, the documentation of a clear trigger was essential for the diagnosis of T2MI. Coronary angiography was not mandatory for a diagnosis of T1MI to limit the effect of selection bias due to clinical referral to coronary angiography. Also, as indicated in the fourth universal definition of MI, different etiologies of acute cardiomyocyte injury such as myocarditis, takotsubo syndrome, and acute heart failure were adjudicated as other cardiac pathologies, distinct from T1MI and T2MI.

\subsection{Blood Sampling and Laboratory Methods}

Blood samples for the determination of hs-cTn were collected at presentation to the $\mathrm{ED}$ and 1 and $2 \mathrm{~h}$ after presentation. After centrifugation, samples were frozen at $-80{ }^{\circ} \mathrm{C}$ until assayed in a blinded fashion in a dedicated core laboratory.

The Roche hs-cTnT assays used the Elecsys 2010 system (Roche Diagnostics, Rotkreuz, Switzerland), with a limit of detection (LoD) of $5 \mathrm{ng} / \mathrm{L}$, a 99th-percentile cut-off point of $14 \mathrm{ng} / \mathrm{L}$, and a coefficient of variation (CV) of less than $10 \%$ at $13 \mathrm{ng} / \mathrm{L}$ [13-15].

The Abbott Architect hs-cTnI assay used was the ARCHITECT High-Sensitivity STAT Troponin I assay (Abbott Laboratories, Abbott Park, IL, USA). The Abbott Architect hs-cTnI assay was performed with the use of the Architect system with a LoD of $1.9 \mathrm{ng} / \mathrm{L}$ and a 99th percentile cut-off point of $26.2 \mathrm{ng} / \mathrm{L}$ with a corresponding CV of $<5 \%$ [16].

\subsection{Calculation of the Neumann Score}

The clinical risk score was applied as recommended [10]. In brief, the Neumann Score included female sex, no radiating chest pain, and a baseline hs-cTnI concentration $\leq 40.8 \mathrm{ng} / \mathrm{L}$ and assigned 1 point per variable, obtaining a total score ranging from 0 to 3 points (Supplementary Table S2). The predicted probability of having T2MI is given by

$$
p=\frac{1}{1+e^{-(\text {Intercept }+ \text { Points })}}
$$

\subsection{Neumann Risk Model}

The Neumann Score was derived from a multivariable logistic model by conversion of the regression coefficients (beta coefficients) to a point-based score. Physicians will use the score for decision-making, therefore performance measurements of the score, and not the risk model, are of main importance. However, for better insight, we provide calibration plots for the risk model, as well as for the point-based score. Calibration plots using beta coefficients for calculating the predicted probability can be found in Supplementary Figures S1 and S2.

The probability of T2MI in individual patients is given by

$$
p=\frac{1}{1+e^{-\left(\beta_{0}+\beta_{1} \times \text { predictor }_{1}+\ldots+\beta_{n} \times \text { predictor }_{n}\right) \prime}}
$$

where $p$ stands for the predicted probability, $\beta_{0}$ for the intercept, and $\beta_{n}$ for the coefficients of the risk variables.

\subsection{Objective}

The primary objective was to evaluate the model performance of the Neumann Score.

\subsection{Statistical Analysis}

Continuous variables are presented as medians (interquartile range (IQR)); categorical variables as numbers and percentages. Differences in baseline characteristics between patients with T1MI and T2MI were assessed using the Mann-Whitney $U$ test for continuous variables and the Pearson Chi-square test for categorical variables. Confidence intervals (CI) of proportions were computed as appropriate [17].

The Neumann Score was externally validated using methods described previously $[18,19]$. To study the performance of the Neumann Score in our validation cohort, we assessed 
its discrimination and calibration. Discrimination is the ability of the score or model to discriminate patients with T2MI from patients without T2MI. Score discrimination was quantified with the area under the curve (AUC), which is equal to the concordance statistics (c-statistic) for a dichotomous outcome variable [20]. Calibration refers to the agreement between the predicted risk and the observed frequencies of T2MI. The model's calibration was graphically assessed through a calibration plot. The calibration plot and its parameters (intercept and slope) were first estimated without adjustment. The score was then recalibrated by adjustment of the intercept (adjustment for baseline risk) [18].

To extend the Neumann Score, multivariable logistic regression analysis was used to determine whether adding different clinical variables to the Neumann Score variables improved model performance. The Neumann Score variables were entered as a score with fixed coefficients rather than as separate variables to prevent the original Neumann Score coefficients to be adjusted in the presence of the new variables, hence modifying the whole risk score. Clinical variables were selected based on feasibility, clinical meaningfulness, and current guidelines. Stepwise selection of additional predictors was used to evaluate potential predictors which were not included in the original model [21]. As the objective was to improve model performance, Brier Score, AUC, and $p$ value of the Hosmer-Lemeshow statistic were assessed for deciding the best final model. Once the final model was chosen, the regression coefficients of the new variables were transformed into a point-based score and added to the Neumann Score, resulting in the Extended Neumann Score.

We assessed improvement in model performance by calculating the change in the AUC, the relative integrated discrimination improvement (IDI), the net reclassification improvement (NRI), and the net benefit in the form of decision curve analysis (DCA), as recommended by the TRIPOD statement [11]. Relative IDI expresses the improvement in T2MI predicted probability on a percentage scale for the Neumann Score and the Extended Neumann Score [22]. The NRI quantifies the ability of the Extended Neumann Score to reclassify patients by counting how many patients with T2MI were reclassified to a higher probability category and how many without T2MI (T1MI) were reclassified to a lower probability category in the Score. We present results for the 2 NRI versions, additive and absolute NRI [23]. Net benefit is a measure that compares benefits and harms. Briefly, it is the difference between the proportion of true positives and the proportion of false positives weighted by the odds of the selected threshold. At any given threshold, the model with the highest net benefit is the preferred model. Net benefit was analyzed using decision curve analysis comparing the Neumann Score with the Extended Neumann Score [24]. Confidence Intervals of AUCs and $p$-values for comparison of AUCs were calculated according to DeLong [25]. Calibration of the extended model was also assessed.

As a performance measure, we report the true positive rate (TPR) and the positive predictive value (PPV) for each point score of the Neumann and Extended Neumann Scores. The PPV is numerically equal to a patient's post-test probability, i.e., the probability of actually having T2MI when diagnosed as such. The TPR (also known as sensitivity) measures the percentage of true T2MI cases correctly detected by the diagnostic test (in this case, the risk score). Therefore, a good diagnostic test has a high PPV, as well as a high TPR. The rule-in performance is visualized by the TPR and the PPV versus the point-based score. The confidence interval for the TPR and PPV was computed using the Wilsons method [26].

We did not perform formal sample size calculations because the cohort study is an ongoing study. Also, there are no generally accepted approaches to estimate the sample size requirements for external validation studies of risk prediction models [11]. Some have suggested having a minimum of 100 events (type 2 MI, in this case) for external validation of clinical prediction rules [27]. Our sample and the number of events far exceeded all approaches for determining samples sizes and, therefore, were expected to provide very robust estimates.

All hypothesis testing was two-tailed, and $p$-values $<0.05$ were considered statistically significant. All statistical analyses were performed using STATA, version 15.1 (Stata Corp, 
College Station, TX, USA), and R, version 3.6.3 (R foundation for Statistical Computing, Free Software Foundation, Boston, MA, USA).

\section{Results}

\subsection{Patient Characteristics}

From April 2006 to April 2018, 6684 patients were recruited. Among them, 1106 (17\%) patients with an adjudicated final diagnosis of non- (N) STEMI, $27(2 \%)$ were excluded from this study because they lacked at least one variable for the Neumann Score calculation. A total of $1079(16 \%)$ patients were eligible for the primary analysis. Of these, $242(22 \%)$ had T2MI (Figure 1). The median patient age was 70 years (IQR 59-79). T2MI and T1MI patients were comparable in many baseline characteristics (Table 1). However, T2MI patients were more often female ( $36 \%$ vs. $26 \%)$, more likely to present with left bundle branch block ( $10 \%$ vs. $6 \%)$ or a higher heart rate ( 90 vs. 76$)$, less often had previous MI ( $27 \%$ vs. $34 \%)$, previous percutaneous coronary intervention (PCI) $(24 \%$ vs. $32 \%)$, and presented with a lower eGFR ( $69 \mathrm{vs} .75 \mathrm{~mL} / \mathrm{min} / \mathrm{m}^{2}$ ) and lower systolic blood pressure (134 vs. $145 \mathrm{mmHg}$ ). The hs-cTnI concentration at presentation was significantly lower in T2MI compared to T1MI patients ( $23 \mathrm{vs} .114 \mathrm{ng} / \mathrm{L}$ ). The most common causes of T2MI (covering $88 \%$ of all cases) were arrhythmias, hypertension, anemia, hypoxemia, and coronary spasm (Supplementary Table S3).

Table 1. Baseline Characteristics in patients with AMI, T1MI and T2MI.

\begin{tabular}{|c|c|c|c|c|}
\hline Baseline Characteristics & All AMI Patients $(n=1079)$ & T1MI $(n=837)$ & T2MI $(n=242)$ & $p$ Value \\
\hline Age, year & $70.0(59.0,79.0)$ & $70.0(58.0,79.0)$ & $72.0(60.0,80.0)$ & 0.33 \\
\hline Female & $307(28.5 \%)$ & $220(26.3 \%)$ & $87(36.0 \%)$ & 0.003 \\
\hline \multicolumn{5}{|l|}{ Cardiovascular risk factors, $n(\%)$} \\
\hline Hypertension & $832(77.1 \%)$ & $645(77.1 \%)$ & $187(77.3 \%)$ & 0.94 \\
\hline Hypercholesterolemia & $671(62.2 \%)$ & $543(64.9 \%)$ & $128(52.9 \%)$ & $<0.001$ \\
\hline Diabetes mellitus & $288(26.7 \%)$ & $225(26.9 \%)$ & $63(26.0 \%)$ & 0.79 \\
\hline Current smoker & $251(23.3 \%)$ & $207(24.7 \%)$ & $44(18.2 \%)$ & 0.034 \\
\hline Former smoker & $438(40.6 \%)$ & $343(41.0 \%)$ & $95(39.3 \%)$ & 0.63 \\
\hline \multicolumn{5}{|l|}{ History, $n(\%)$} \\
\hline Coronary artery disease & $480(44.5 \%)$ & $380(45.4 \%)$ & $100(41.3 \%)$ & 0.26 \\
\hline Previous MI & $353(32.7 \%)$ & $287(34.3 \%)$ & $66(27.3 \%)$ & 0.04 \\
\hline Previous CABG & $160(14.8 \%)$ & $128(15.3 \%)$ & $32(13.2 \%)$ & 0.42 \\
\hline Previous PCI & $329(30.5 \%)$ & $270(32.3 \%)$ & $59(24.4 \%)$ & 0.019 \\
\hline Peripheral artery disease & $122(11.3 \%)$ & $98(11.7 \%)$ & $24(9.9 \%)$ & 0.44 \\
\hline Previous stroke & $97(9.0 \%)$ & $78(9.3 \%)$ & $19(7.9 \%)$ & 0.48 \\
\hline \multicolumn{5}{|l|}{ ECG findings, $n(\%)$} \\
\hline Left bundle-branch block & $73(6.8 \%)$ & $49(5.9 \%)$ & $24(9.9 \%)$ & 0.027 \\
\hline ST-segment depression & $111(10.3 \%)$ & $79(9.4 \%)$ & $32(13.2 \%)$ & 0.088 \\
\hline T-wave inversion & $243(22.5 \%)$ & $196(23.4 \%)$ & $47(19.4 \%)$ & 0.19 \\
\hline \multicolumn{5}{|l|}{ Laboratory findings } \\
\hline hs-TnI on admission (ng/L) & $76.3(20.3,423.8)$ & $114.0(28.0,576.1)$ & $23.1(10.0,83.1)$ & $<0.001$ \\
\hline Haemoglobin (g/dL) & $141.0(127.0,153.0)$ & $142.0(127.0,153.0)$ & $140.0(125.0,152.0)$ & 0.15 \\
\hline $\mathrm{eGFR},\left(\mathrm{mLmin} / \mathrm{m}^{2}\right)$ & $73.6(57.8,92.2)$ & $75.3(60.0,94.1)$ & $69.2(52.1,88.6)$ & $<0.001$ \\
\hline \multicolumn{5}{|l|}{ Vital signs on admission } \\
\hline Systolic blood pressure (mmHg) & $141.0(125.0,160.0)$ & $145.0(128.0,161.0)$ & $134.0(116.0,153.0)$ & $<0.001$ \\
\hline Heart rate (bpm) & $78.0(68.0,92.0)$ & $76.0(66.0,88.0)$ & $89.5(73.0,120.0)$ & $<0.001$ \\
\hline Respiratory rate (per minute) & $16.0(14.0,20.0)$ & $16.0(14.0,20.0)$ & $16.0(14.0,20.0)$ & 0.76 \\
\hline $\mathrm{SaO}_{2}(\%)$ & $98.0(96.0,99.0)$ & $98.0(96.0,99.0)$ & $98.0(96.0,99.0)$ & 0.71 \\
\hline \multicolumn{5}{|l|}{ Chest pain characteristics } \\
\hline No radiating chest pain & $388(36.0 \%)$ & $280(33.5 \%)$ & $108(44.6 \%)$ & 0.001 \\
\hline
\end{tabular}


Table 1. Cont.

\begin{tabular}{|c|c|c|c|c|}
\hline Baseline Characteristics & All AMI Patients $(n=1079)$ & T1MI $(n=837)$ & T2MI $(n=242)$ & $p$ Value \\
\hline Admission medication & & & & \\
\hline Antiaggregant (aspirin + Clopidogrel) & $549(50.9 \%)$ & $440(52.6 \%)$ & $109(45.0 \%)$ & 0.039 \\
\hline Warfarin (Vitamin K antagonist) & $125(11.6 \%)$ & $81(9.7 \%)$ & $44(18.2 \%)$ & $<0.001$ \\
\hline Beta-blocker & $460(42.6 \%)$ & $342(40.9 \%)$ & $118(48.8 \%)$ & 0.029 \\
\hline Statin & $481(44.6 \%)$ & $375(44.8 \%)$ & $106(43.8 \%)$ & 0.78 \\
\hline ACEIs/ARBs & $574(53.2 \%)$ & $443(52.9 \%)$ & $131(54.1 \%)$ & 0.74 \\
\hline Calcium antagonists & $220(20.4 \%)$ & $174(20.8 \%)$ & $46(19.0 \%)$ & 0.54 \\
\hline Nitrates & $165(15.3 \%)$ & $139(16.6 \%)$ & $26(10.7 \%)$ & 0.026 \\
\hline
\end{tabular}

Table Legend: Displayed are the baseline characteristics for all AMI patients and stratification by AMI type. The $p$-value is given for the comparison between T1MI and T2MI patients. MI, myocardial infarction; T1MI, type 1 myocardial infarction; T2MI, type 2 myocardial infarction; $\mathrm{CABG}$, coronary artery bypass graft; $\mathrm{PCI}$, percutaneous coronary intervention; eGFR, estimated glomerular filtration rate; $\mathrm{SaO}$, oxygen saturation; $\mathrm{ACEI} / \mathrm{ARB}$, angiotensin converting enzyme inhibitor/angiotensin receptor blocker, ECG, electrocardiogram.

\subsection{External Validation of the Neumann Score}

The Neumann Score distribution and its relationship with the observed probability of T2MI is shown in Figure 2. The diagnostic accuracy of the Neumann Score was moderate, with a c-statistic of 0.67 (95\% CI 0.64-0.71). The calibration plot showed a moderate agreement between the predicted probabilities of T2MI according to the Neumann Score and the observed frequencies (Figure 3A). The predicted probabilities were underestimated for the lowest risk groups (score $0-2$ ) assessed by visual inspection and confirmed with a calibration intercept of 0.44 (CI, 0.29-0.59). An intercept $>0$ indicates that the score's predicted probabilities in the validation set are systematically too low. Agreement for the highest risk group (Score of 3) was optimal. The calibration slope was 0.79 (CI, 0.61-0.97). The Hosmer-Lemeshow test for external validation yielded a $p$-value $<0.001$, indicating suboptimal agreement.

A

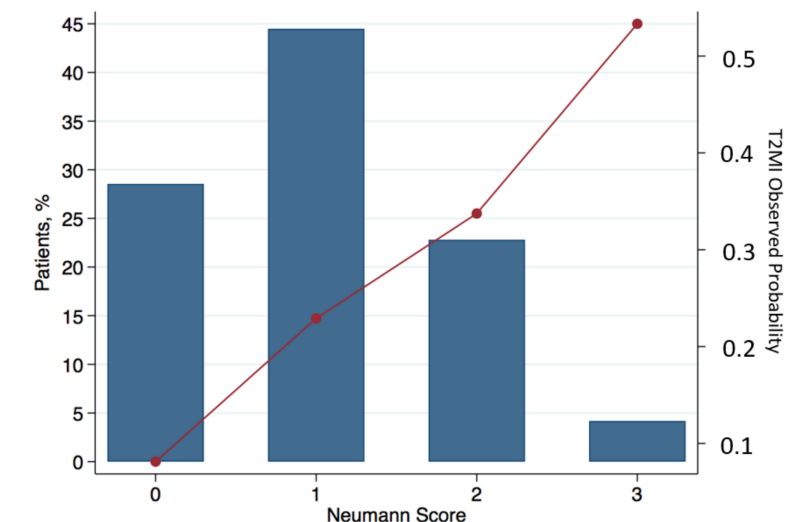

B

\begin{tabular}{|cccccc|}
\hline \multicolumn{7}{|c|}{ Neumann Score } \\
\hline \multicolumn{7}{|c|}{1} & 2 & 3 & Total \\
\hline T1MI, $\mathbf{n}$ & 283 & 370 & 163 & 21 & 837 \\
\hline$\%$ & $(33.81)$ & $(44.21)$ & $(19.47)$ & $(2.51)$ & $(100)$ \\
\hline T2MI, $\mathbf{n}$ & 25 & 110 & 83 & 24 & 242 \\
\hline$\%$ & $(10.33)$ & $(45.45)$ & $(34.30)$ & $(9.92)$ & $(100)$ \\
\hline Total, $\mathbf{n}$ & 308 & 480 & 246 & 45 & 1079 \\
\hline$\%$ & $(28.54)$ & $(44.49)$ & $(22.80)$ & $(4.17)$ & $(100)$ \\
\hline
\end{tabular}

Figure 2. Neumann risk score distribution and relationship with the observed probability of T2MI for each score. (A) Distribution of the calculated Neumann risk score and its relationship with the observed probability of T2MI in all 1079 patients. (B) Total number of AMI patients for each score and patient stratification for T1MI and T2MI. Percentages show the number of patients in each group with respect to the total number of T1MI or T2MI patients (left Y-axis). For calculating the observed probability of T2MI in the validation cohort, the reader only needs to divide the number of T2MI/all patients for the desired score (e.g., for a 3-point score, the observed probability of T2MI would be $24 / 45=53.3 \%$ ). The observed probability for each point score appears in the right Y-axis. 

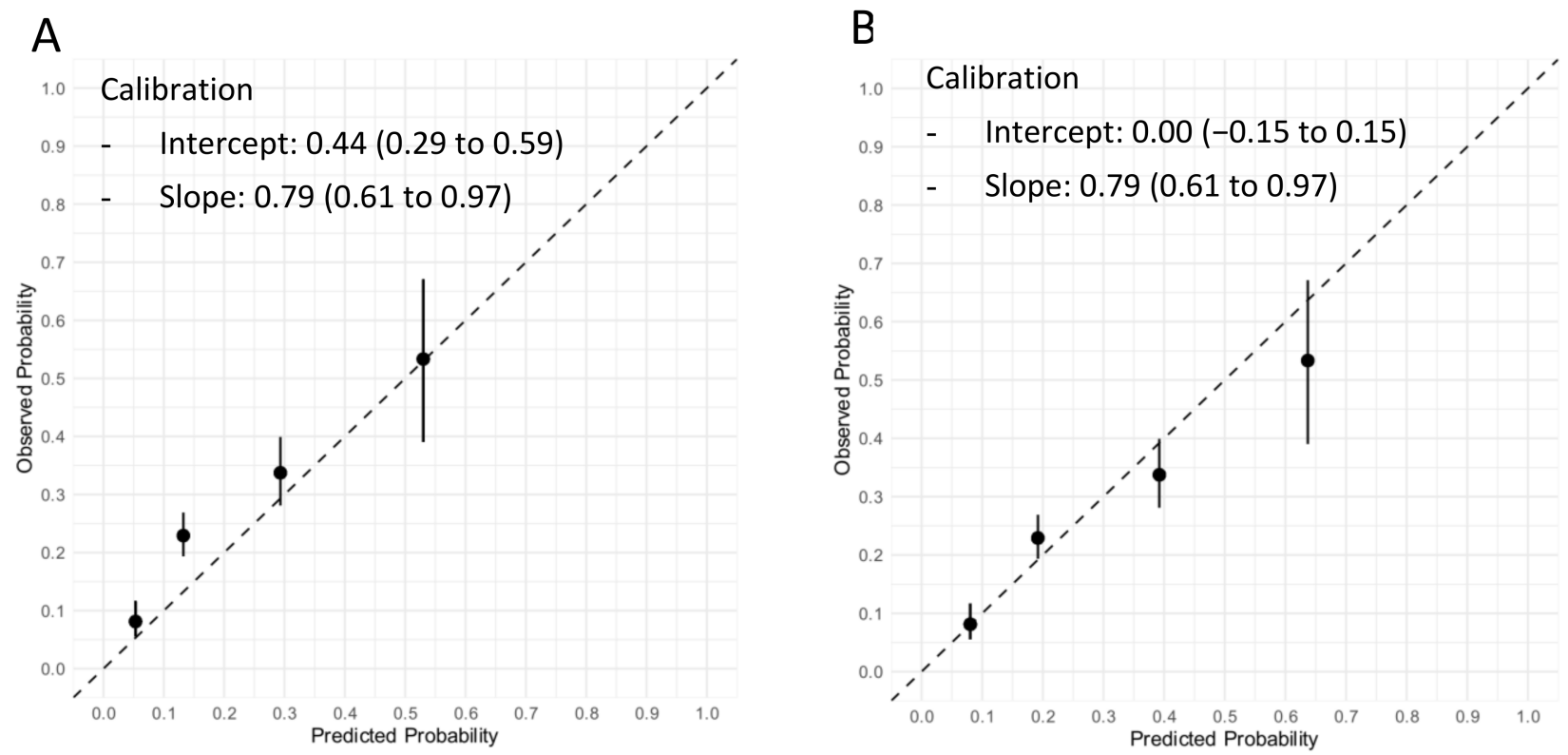

Figure 3. Calibration plot of the original and recalibrated Neumann Score tested in the validation cohort. Assessment of goodness of fit. (A) Calibration plot of the Neumann Score tested in the validation cohort. The highest predicted probability $(0.53)$ is obtained with a score of 3 . An intercept $>0$ indicates that the score's predicted probabilities in the validation cohort are systematically too high. (B) Calibration plot of the recalibrated Neumann Score tested in the validation cohort. The highest predicted probability (0.64) is obtained with a score of 3 . The agreement between predicted and observed probability appears improved for low risk Scores. An overestimation of the predicted probability can now be observed for the highest score (3). Perfect calibration is represented by the dotted line through the origin. Whiskers indicate $95 \%$ CI's.

To adjust for different baseline risks between the derivation cohort and our external validation cohort, the Neumann Score was recalibrated. The recalibrated Neumann Score showed improved calibration for the lower risk groups (risk scores $0-2$ ), with correction of the underestimation for these groups indicated by the calibration points appearing nearer to the optimal line $\left(45^{\circ}\right.$ dotted line). However, a modest overestimation for the highest risk group (risk score 3) was now observed (Figure 3B). With regard to the overall calibration, the calibration slope was maintained (0.79 (CI, 0.61-0.97)). After recalibration, the Hosmer-Lemeshow test yielded a $p$-value of 0.20 .

The calibration plots of the prediction model using original beta regression coefficients instead of the point-based score can be found in Supplementary Figure S1.

\subsection{Extension of the Neumann Score}

Multivariable logistic regression analysis showed that extending the Neumann Score by adding the parameter "heart rate 120 beats per minute" significantly improved the diagnostic accuracy assessed by AUC, Brier Score, and Hosmer-Lemeshow test (Table 2). This variable was weighted 2 points if present and 0 points if absent. The Extended Neumann Score, its distribution, and its relationship with the observed frequency of T2MI is shown in Figure 4, central illustration.

Addition of heart rate to the Neumann Score significantly increased the diagnostic accuracy of the Score (c-statistic 0.73; 95\% CI 0.70-0.76, $p<0.001$, Figure 5). Improvement in model performance was also confirmed by improvement in the IDI and NRI. The relative improvement in IDI was $9.7 \%$ for predicting T2MI, while the additive NRI was 14.8, and the absolute NRI was 3.2\%, favoring the Extended Neumann Score. (Supplementary Table S4). When assessing the additive NRI individually, we obtained better reclassification of patients with T2MI $(15.3 \%)$ and not worse reclassification of patients without T2MI (incorrect reclassification by $0.1 \%$ ). In other words, the Extended Neumann Score improved the diagnosis of patients with T2MI without worsening the diagnosis of patients with T1MI. 
Table 2. Multivariable logistic regression analysis for the diagnosis of T2MI.

\begin{tabular}{|c|c|c|c|c|c|c|}
\hline \multicolumn{7}{|c|}{ Multivariable Analysis } \\
\hline Variables & Beta Coefficient (95\% CI) & OR (95\% CI) & $p$-Value & Brier & AUC & hl \\
\hline Age $<70$ years & $0.119(-0.187-0.423)$ & $1.126(0.829-1.526)$ & 0.446 & 0.163 & 0.671 & 0.138 \\
\hline Heart rate $>120 / \mathrm{min}$ & $2.472(1.935-3.038)$ & $11.842(6.927-20.866)$ & $<0.001$ & 0.145 & 0.732 & 0.239 \\
\hline Systolic blood pressure $>160 \mathrm{mmHg}$ & $-0.350(-0.730-0.016)$ & $0.705(0.482-1.017)$ & 0.066 & 0.163 & 0.68 & 0.142 \\
\hline No previous coronary artery disease & $0.218(-0.088-0.527)$ & $1.244(0.915-1.694)$ & 0.165 & 0.162 & 0.673 & 0.047 \\
\hline No previous myocardial infarction & $0.434(0.102-0.774)$ & $1.543(1.108-2.167)$ & 0.011 & 0.161 & 0.679 & 0.038 \\
\hline No pathological ECG changes & $-0.155(-0.459-0.149)$ & $0.857(0.632-1.161)$ & 0.318 & 0.163 & 0.675 & 0.137 \\
\hline $\mathrm{eGFR} \leq 30 \mathrm{~mL} / \mathrm{min} / 1.73 \mathrm{~m}^{2}$ & $0.557(-0.133-1.214)$ & $1.746(0.876-3.365)$ & 0.103 & 0.163 & 0.677 & 0.263 \\
\hline Heart Rate $>120 / \mathrm{min}+$ no previous MI & & & & 0.145 & 0.736 & 0.066 \\
\hline
\end{tabular}

Table Legend: Provided is the multivariable logistic regression for the diagnosis of T2MI. Female sex, no radiating chest pain, and baseline hs-TnI were fixed in the regression. The predictors were tested one by one. As the objective was to improve model performance, Brier Score, AUC (the area under the curve), and $p$ value of the Hosmer-Lemeshow statistics were assessed for deciding the best final model. Heart rate $>120 \mathrm{bpm}$ showed the highest model performance. No improvement to either the area under the curve or the Brier Score was observed by including any additional variables. No improvement to either AUC or the Brier Score was observed when adding the parameter "no previous myocardial infarction" to "heart rate $>120$ beats per minute", while the model's goodness of fit decreased ( $p$ value for Hosmer-Lemeshow test $=0.066$ ). Thus, only the predictor "heart rate $>120$ beats per minute" was introduced in the Neumann Score OR, odds ratio; hl: Hosmer-Lemeshow; CI, confidence interval; ECG, electrocardiogram.

A

\begin{tabular}{lcc}
\hline \multicolumn{3}{c}{ Extended Neumann Score } \\
\hline Characteristic & Categories & Points \\
\hline Female Sex & No & 0 \\
& Yes & 1 \\
\hline Radiating Chest Pain & No & 1 \\
& Yes & 0 \\
\hline Baseline Troponin & hs-Tnl $\leq 40.8 \mathrm{ng} / \mathrm{L}$ & 1 \\
& hs-Tnl $>40.8 \mathrm{ng} / \mathrm{L}$ & 0 \\
\hline Heart rate $>\mathbf{1 2 0}$ bpm & No & 0 \\
& Yes & 2 \\
\hline
\end{tabular}

B

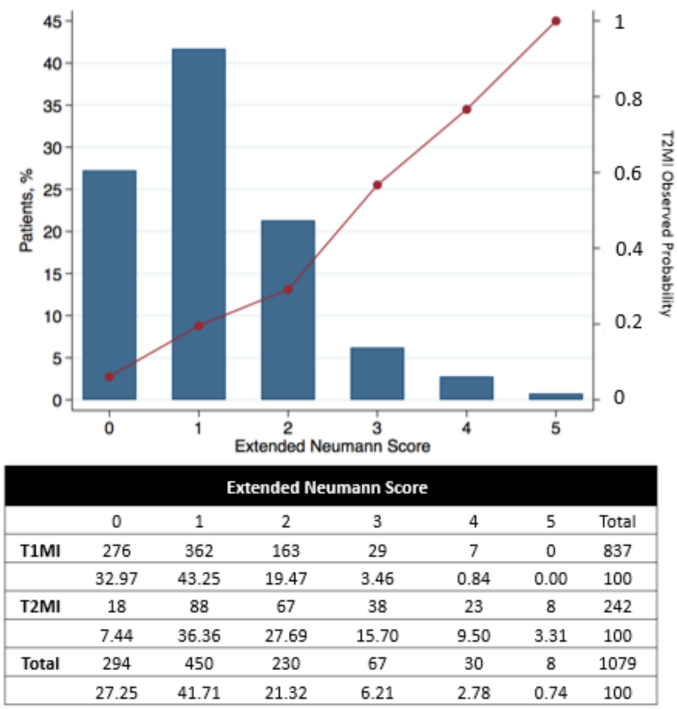

Figure 4. Extended Neumann Score, its distribution, and its relationship with the observed probability of T2MI for each Score. (A) Extended Neumann risk score. (B) Distribution of the calculated Extended Neumann risk score and its relationship with the observed probability of T2MI for all 1079 patients. (C) Total number of AMI patients for each score and stratification for T1 and T2MI. Percentages show the number of patients in each group with respect to the total number of T1 or T2MI patients (left Y-axis). For calculating the observed probability of T2MI in the validation cohort, the reader only needs to divide the number of T2MI/all patients for the desired score (e.g., for a 3-point score, the observed probability of T2MI would be $38 / 67=56.7 \%$ ). The observed probability for each point score appears in the right $\mathrm{Y}$-axis of $(\mathbf{B})$.

The decision curve analysis showed improvement in net benefit with the Extended Neumann Score at nearly any threshold probability for diagnosing T2MI (Supplementary Figure S2). The DCA clearly separated from a threshold probability of 0.2 onwards. Given the relative risks of missing a T1MI diagnosis compared to the harms of coronary angiography, we would consider it reasonable for any patient or doctor to demand at least a $70 \%$ risk of having T2MI before accepting delayed or no coronary angiography. The net benefit across the high probability threshold range (above $70 \%$ ) was of approximately $2 \%$. When translated to interventions avoided, the Extended Neumann Score would reduce 
unnecessary angiographies in comparison to the Neumann Score by an additional $2 \%$ for a threshold probability of $70 \%$ and by an additional $3 \%$ for a threshold probability of $50 \%$.

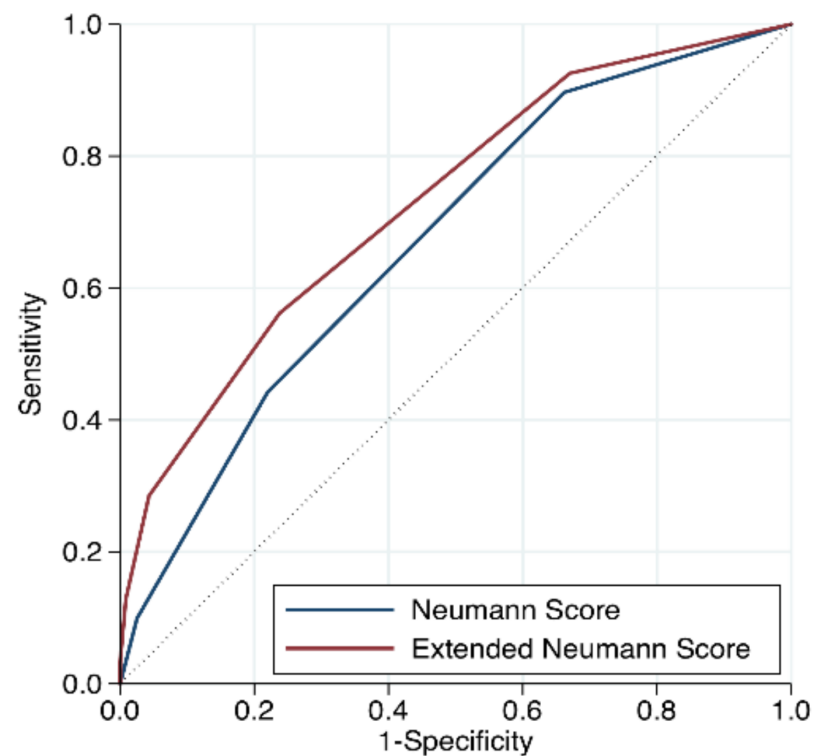

Figure 5. Receiver operating characteristic curve to diagnose type 2 MI for the Neumann and Extended Neumann risk Scores.

The calibration plot of the Extended Neumann Score showed strong agreement between predicted and observed proportions, obtaining a near optimal slope of 0.91 (CI, 0.75-1.07) and an ideal intercept (0.00 (CI, - 0.16 to 0.16), Figure 6). A non-significant Hosmer-Lemeshow test $(p=0.51)$ confirmed the goodness-of-fit. The calibration plots of the prediction model using original beta regression coefficients instead of the point-based score can be found in Supplementary Figure S3.

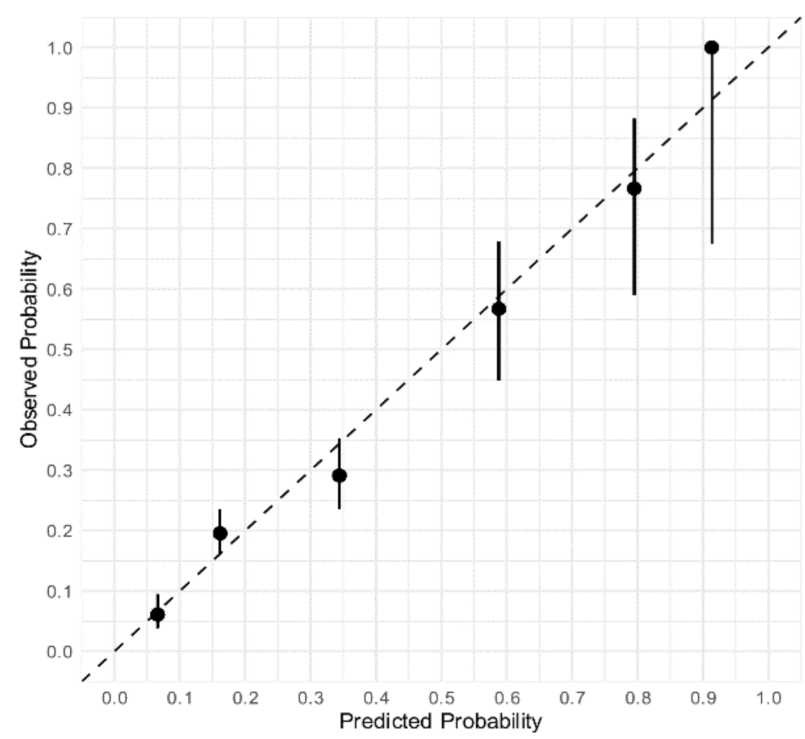

Figure 6. Calibration plot of the extended Neumann Score tested in the validation cohort. Assessment of goodness of fit. Calibration plot of the prediction Score with the extended Neumann risk groups. The highest predicted probability (1.0) is obtained with a score of 5. A nearly optimal calibration slope was achieved. The calibration plot shows good agreement overall, assessed by the proximity of the six scores (0 to 5). Confidence interval increases as sample size reduces in the score groups. Perfect calibration is represented by the dotted line through the origin. Whiskers indicate $95 \%$ CIs. 


\subsection{Diagnostic Performance of the Neumann and Extended Neumann Scores}

The diagnostic performance of both risk scores is displayed in Supplementary Table S5. Classification performance for ruling in T2MI is shown in Figure 7.
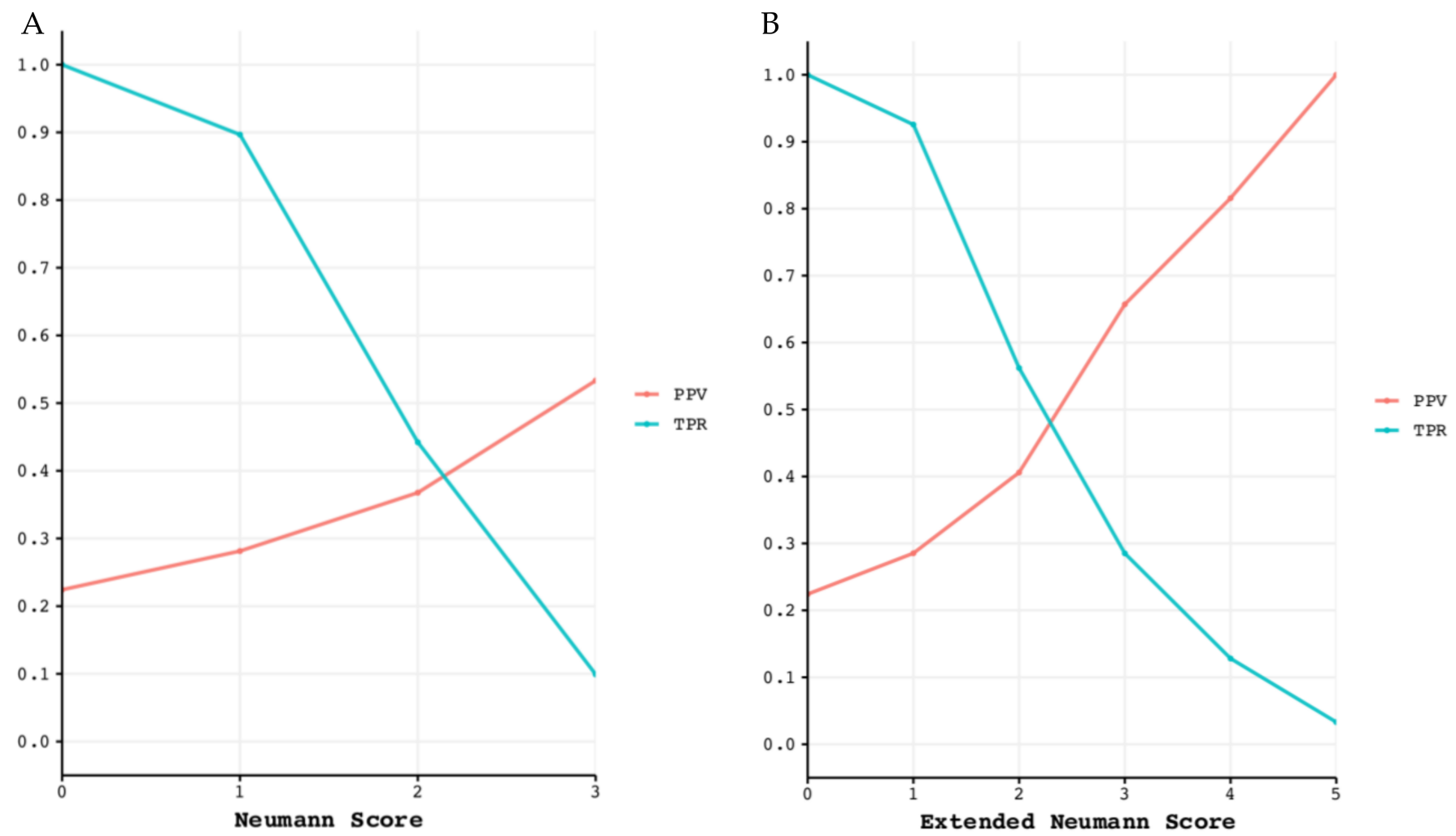

Figure 7. Classification performance for ruling in T2MI. Positive predictive value (PPV) and true positive rate (TPR) for each point of the Neumann (left) and Extended Neumann Scores (right). (A) The maximum reached PPV with the Neuman Score is $53 \%$, meaning that $53 \%$ of patients scoring 3 points are correctly diagnosed with T2MI. For a Score of 3 , the TPR is $10 \%$, meaning that $10 \%$ of all T2MI patients have a score of 3. (B) The maximum PPV reached with the Extended Neumann Score is $100 \%$, meaning that all patients scoring 5 points are correctly diagnosed with T2MI. For a score of 5 , the TPR is $3.3 \%$, meaning that $3.3 \%$ of all T2MI patients have a score of 5 .

\section{Discussion}

This large multicenter diagnostic study aimed to contribute to overcoming the clinical uncertainty about the diagnosis T2MI and T1MI by externally validating and extending the recently derived Neumann Score [10]. We report six major findings:

First, in this validation cohort, a Neumann Score (female sex, absence of radiating chest pain, and hs-cTnI-Architect $<40.8 \mathrm{ng} / \mathrm{L}$ ) of 3 points (the highest achievable score) was associated with a $53 \%$ observed probability of having T2MI, with only $10 \%$ of T2MI patients reaching a score of 3 (Figure 2). Second, the Neumann Score showed moderate discrimination (AUC $0.67,95 \% \mathrm{CI} 0.64-0.71$ ), comparable to that observed in the Hamburg single-center derivation cohort (AUC 0.71, 95\%CI 0.67-0.79) [10]. Third, the calibration plot showed a positive intercept, which indicated an overall underestimation for predicting T2MI risk. Fourth, extending the Neumann Score by adding heart rate at presentation $>120 / \mathrm{min}$ as an additional binary variable increased the diagnostic accuracy to 0.73 (95\%CI $0.70-0.76, p<0.001)$. Improvement in model performance was also confirmed by improvement in IDI, NRI, and DCA. Fifth, the Extended Neumann Score showed good overall calibration (Hosmer-Lemeshow test, $p=0.51$ ). A Visual examination of the calibration plot demonstrated strong agreement between the predicted probabilities and the observed proportions of T2MI along all score groups. Sixth, 5 points (highest score) for the Extended Neumann Score corresponded to $100 \%$ observed probability of T2MI, 
although a minority of patients reached it (3\%). When a score is derived with the aim to diagnose a disease which has primarily a non-invasive therapy (T2MI) in contrast to other possible diagnoses which should receive an invasive procedure (coronary angiography, T1MI), the score should be favored for a very high PPV (high safety) and reduce the number of false positives (false positive $=$ the patient has T1MI but is classified as a T2MI patient by the score and does not receive coronary angiography). Therefore, the Extended Neumann Score represents a clinically meaningful improvement versus the original score [10]. However, due to the only moderate performance of both scores, their clinical implementation may be of limited usefulness for the clinician. Further improvement is necessary, possibly by adding additional cardiovascular biomarkers and other criteria. Additionally, the evaluation of a score to predict short- and long-term outcomes in T2MI and T1MI patients is a major clinical need for further improvement of care, especially for T2MI.

Still, the low percentage of correctly classified T2MI patients indicates that in many patients, invasive or non-invasive anatomical and/or functional cardiac imaging including coronary angiography will remain necessary for the accurate differentiation of T2MI with respect to T1MI [2]. Particularly when deriving and validating prediction rules for T2MI, it is mandatory to strictly adhere to the universal definition of MI [2] and not lump patients with cardiomyocyte injury of unknown or miscellaneous cause such as acute heart failure, Takotsubo syndrome, and myocarditis also into the group of T2MI. This error could overestimate the predicted probability of having T2MI.

Several limitations should be considered when interpreting these findings. First, this study was conducted in patients presenting to the ED. We cannot comment on the performance of the Extended Neumann Score in other clinical settings including the perioperative setting and critically ill patients. Second, although we used a very stringent methodology to adjudicate T1MI and T2MI including central adjudication by experienced cardiologists using cardiac imaging and serial measurements of hs-cTn, we may still have misclassified a small number of patients [2]. This could have led to an underestimation of the true accuracy of the Neumann Score and the Extended Neumann Score. Third, the Neumann Score includes hs-cTnI measured with one specific analyzer. Future studies need to define the optimal hs-cTnI/T cut-offs for other clinically used hs-cTnI/T assays. Fourth, we cannot generalize these findings to patients with terminal kidney failure requiring dialysis, since these patients were excluded from this study.

\section{Conclusions}

In conclusion, external validation of the Neumann Score showed moderate discrimination and suboptimal calibration. Extending the Neumann Score by adding the parameter heart rate $>120 /$ min significantly improved the model's performance.

Supplementary Materials: The following are available online at https: / /www.mdpi.com/2077-0 383/10/6/1264/s1, Figure S1A: Calibration plot of the Neumann prediction model, Figure S1B: Calibration plot of the recalibrated Neumann prediction model, Figure S2: Decision curve analysis of net benefit for heart rate addition (Extended Neumann Score) to the Neumann Score for diagnosis of T2MI, Figure S3: Calibration plot of the Extended Neumann prediction model, Table S1: TRIPOD checklist, Table S2: Original Neumann Score, Table S3: T2MI underlying main triggers, Table S4: Risk reclassification for diagnosis of T2MI based on the addition of heart rate (Extended Score) to the Neumann Score, Table S5A: Diagnostic performance of the Neumann Score, Table S5B: Diagnostic performance of the Extended Neumann Score.

Author Contributions: Conceptualization, T.N., P.L.-A., J.B., R.T. and C.M.; methodology, T.N., P.L.-A., J.B., I.S. and C.M.; software, T.N., P.L.-A., J.B., I.S. and C.M.; validation, T.N., P.L.-A., J.B., I.S., M.R.G., I.H., K.W., D.W., L.K., A.P., D.M.G., C.P., N.G., P.H., S.F., A.B., Ò.M., F.J.M.-S., D.K.(Damian Kawecki), D.K. (Dagmar Keller), R.W., R.T. and C.M.; formal analysis, T.N., P.L.-A., J.B., I.S. and C.M.; investigation, C.M.; resources, C.M.; data curation, T.N., P.L.-A., J.B., I.S. and C.M.; writing-original draft preparation, T.N., P.L.-A., J.B. and C.M.; writing-review and editing, T.N., P.L.-A., J.B. and C.M.; visualization, T.N., P.L.-A., J.B. and C.M.; supervision, T.N., P.L.-A., J.B. and C.M.; project administration, T.N., P.L.-A., J.B. and C.M.; funding acquisition, T.N., P.L.-A., J.B. and C.M. All 
authors have read and agreed to the published version of the manuscript. The sponsors had no role in designing or conducting the study and no role in gathering or analyzing the data or writing the manuscript. The manuscript and its contents have not been published previously and are not being considered for publications elsewhere in whole or in part in any language, including publicly accessible web sites or e-print servers.

Funding: The study was supported by research grants from the Swiss National Science Foundation, the Swiss Heart Foundation, the KTI, the Stiftung für kardiovaskuläre Forschung Basel; Abbott, Beckman-Coulter, Biomerieux, Brahms, Ortho Clinical Diagnostics, Quidel, Roche, Siemens, and Singulex.

Institutional Review Board Statement: The study was conducted according to the guidelines of the Declaration of Helsinki, and approved by the Local Ethics Committee (Ethic Nordwestschweiz, Basel, Switzerland, Number 280/05; date of approval: 28 November 2005.

Informed Consent Statement: Informed consent was obtained from all subjects involved in the study.

Data Availability Statement: Data is available upon request to the corresponding author.

Acknowledgments: We are indebted to the patients who participated in the study and to the emergency department staff as well as the laboratory technicians of all participating sites for their most valuable efforts. We would like to thank the authors from the original manuscript (Neumann and colleagues) for providing us the intercept from their original Neumann Score for graphically assessing the calibration plots. We thank the APACE Investigators, including Tobias Reichlin, Katharina Rentsch, Sandra Mitrovic, Mario Maier, Valentina Troester, Juliane Gehrke, Tania Coscia, Joan Walter, Ana Yufera Sanchez, Jeanne du Fay de Lavallaz, Samyut Shrestha, Beatriz López, Esther Rodriguez Adrada, Piotr Muzyk, Beata Morawiec, Jens Lohrmann, Andreas Buser, Arnold von Eckardstein, Michael Christ, Paul David Ratmann, Patrick Badertscher, Ketina Arslani, Eleni Michou, Tobias Zimmermann, Michael Freese, Nicolas Geigy.

Conflicts of Interest: We disclose that Nestelberger has received research support from the Swiss National Science Foundation (P400PM_191037/1), the Swiss Heart Foundation (FF20079), the Max Cloëtta Foundation, the Margarete und Walter Lichtenstein-Stiftung (3MS1038), and the University Hospital Basel as well as speaker honoraria/consulting honoraria from B.Braun, Siemens, Beckman Coulter, Bayer, Ortho Clinical Diagnostics, and Orion Pharma, outside the submitted work. Lopez-Ayala has received research funding from the Swiss Heart Foundation (FF20079), outside the submitted work. Boeddinghaus has received research grants from the University of Basel and the Division of Internal Medicine, the Swiss Academy of Medical Sciences, the Gottfried and Julia Bangerter-Rhyner-Foundation, and speaker honoraria from Siemens, outside the submitted work. Rubini Gimenez has received research grants from the Swiss Heart Foundation and Swiss National Science Foundation (P400PM_180828) as well as speakers/consulting honoraria from Abbott, Ortho Clinical Diagnostics, Roche, and Siemens, outside the submitted work. Wildi has received research funding from the FAG Basel, and the Julia und Gottfried Bangerter-Rhyner Stiftung, the Prince Charles Hospital Foundation, the CRE Action Fund (NHMRC), the Wesley Medical Research Foundation, and a PhD n UQ scholarship from the University of Queensland, all outside the submitted work. Koechlin has received a research grant from the University of Basel, the Swiss Academy of Medical Sciences, and the Gottfried and Julia Bangerter-Rhyner Foundation, as well as the "Freiwillige Akademische Gesellschaft Basel", outside the submitted work. Gualandro has received consulting honoraria from Roche, outside the submitted work. Martin-Sanchez has received speaker, advisory or consulting fees from Novartis, MSD, Bristol-Myers Squibb, Pfizer, The Medicine Company, Otsuka, Thermo Fisher, Cardiorentis, Sanofi, and research grants from the Spanish Ministry of Health and FEDER, Mapfre, Novartis, Bayer, MSD, Abbott, and Orion-Pharma, outside the submitted work. Twerenbold has received research support from the Swiss National Science Foundation (P300PB167803/1) and speaker honoraria/consulting honoraria from Roche, Abbott, Brahms and Siemens, outside the submitted work. Mueller has received research support from the Swiss National Science Foundation, the Swiss Heart Foundation, the KTI, the Stiftung für kardiovaskuläre Forschung Basel; Abbott, Alere, Astra Zeneca, Beckman Coulter, Biomerieux, Brahms, Roche, Siemens, Singulex, Sphingotec, and the Department of Internal Medicine, University Hospital Basel, as well as speaker honoraria/consulting honoraria from Abbott, Alere, Astra Zeneca, Biomerieux, Boehringer Ingelheim, BMS, Brahms, Cardiorentis, Novartis, Roche, Siemens, and Singulex, outside the submitted work. All other authors declare that they have no conflict of interest with this study. The investigated 
hs-cTn assay were donated by the manufacturer, who had no role in the design of the study, the analysis of the data, the preparation of the manuscript, or the decision to submit the manuscript for publication.

\section{References}

1. Collet, J.-P.; Thiele, H.; Barbato, E.; Barthélémy, O.; Bauersachs, J.; Bhatt, D.L.; Dendale, P.; Dorobantu, M.; Edvardsen, T.; Folliguet, T.; et al. 2020 ESC Guidelines for the management of acute coronary syndromes in patients presenting without persistent ST-segment elevation. Eur. Hear. J. 2020. [CrossRef] [PubMed]

2. Thygesen, K.; Alpert, J.S.; Jaffe, A.S.; Chaitman, B.R.; Bax, J.J.; Morrow, D.A.; White, H.D.; Mickley, H.; Crea, F.; Van de Werf, F.; et al. Fourth Universal Definition of Myocardial Infarction. J. Am. Coll. Cardiol. 2018, 72, 2231-2264. [CrossRef]

3. Sandoval, Y.; Jaffe, A.S. Type 2 Myocardial Infarction. J. Am. Coll. Cardiol. 2019, 73, 1846-1860. [CrossRef]

4. McCarthy, C.P.; Vaduganathan, M.; Januzzi, J.L. Type 2 Myocardial Infarction-Diagnosis, Prognosis, and Treatment. JAMA 2018, 320, 433. [CrossRef]

5. Nestelberger, T.; Boeddinghaus, J.; Badertscher, P.; Twerenbold, R.; Wildi, K.; Breitenbücher, D.; Sabti, Z.; Puelacher, C.; Giménez, M.R.; Kozhuharov, N.; et al. Effect of Definition on Incidence and Prognosis of Type 2 Myocardial Infarction. J. Am. Coll. Cardiol. 2017, 70, 1558-1568. [CrossRef] [PubMed]

6. Chapman, A.R.; Mills, N.L. Refining the Diagnosis of Type 2 Myocardial Infarction. JAMA Cardiol. 2017, 2, 106. [CrossRef]

7. DeFilippis, A.P.; Chapman, A.R.; Mills, N.L.; De Lemos, J.A.; Arbab-Zadeh, A.; Newby, L.K.; Morrow, D.A. Assessment and Treatment of Patients With Type 2 Myocardial Infarction and Acute Nonischemic Myocardial Injury. Circulation 2019, 140, 1661-1678. [CrossRef] [PubMed]

8. Chapman, A.R.; Adamson, P.D.; Shah, A.S.; Anand, A.; Strachan, F.E.; Ferry, A.V.; Ken Lee, K.; Berry, C.; Findlay, I.; Cruikshank, A.; et al. High-Sensitivity Cardiac Troponin and the Universal Definition of Myocardial Infarction. Circulation 2020, 141, 161-171. [CrossRef]

9. Schoepfer, H.; Nestelberger, T.; Boeddinghaus, J.; Twerenbold, R.; Lopez-Ayala, P.; Koechlin, L.; Wussler, D.; Zimmermann, T.; Miro, O.; Martín-Sánchez, J.F.; et al. Effect of a Proposed Modification of the Type 1 and Type 2 Myocardial Infarction Definition on Incidence and Prognosis. Circulation 2020, 142, 2083-2085. [CrossRef] [PubMed]

10. Neumann, J.; Soerensen, N.; Ruebsamen, N.; Ojeda, F.; Renne, T.; Qaderi, V.; Teltrop, E.; Kramer, S.; Quantius, L.; Zeller, T.; et al. Discrimination of Patients with Type 2 Myocardial Infarction. Eur. Hear. J. 2017, 38, 3514-3520. [CrossRef]

11. Collins, G.S.; Reitsma, J.B.; Altman, D.G.; Moons, K.G.M. Transparent Reporting of a multivariable prediction model for Individual Prognosis Or Diagnosis (TRIPOD): The TRIPOD Statement. Ann. Intern. Med. 2015, 162, 55-63. [CrossRef]

12. Levey, A.S.; Coresh, J.; Greene, T.; Stevens, L.A.; Zhang, Y.; Hendriksen, S.; Kusek, J.W.; Van Lente, F. Using Standardized Serum Creatinine Values in the Modification of Diet in Renal Disease Study Equation for Estimating Glomerular Filtration Rate. Ann. Intern. Med. 2006, 145, 247-254. [CrossRef]

13. Reichlin, T.; Hochholzer, W.; Bassetti, S.; Steuer, S.; Stelzig, C.; Hartwiger, S.; Biedert, S.; Schaub, N.; Buerge, C.; Potocki, M.; et al. Early Diagnosis of Myocardial Infarction with Sensitive Cardiac Troponin Assays. New Engl. J. Med. 2009, 361, 858-867. [CrossRef] [PubMed]

14. Stiegler, H.; Fischer, Y.; Vazquez-Jimenez, J.F.; Graf, J.; Filzmaier, K.; Fausten, B.; Janssens, U.; Gressner, A.M.; Kunz, D. Lower cardiac troponin $\mathrm{T}$ and I results in heparin-plasma than in serum. Clin. Chem. 2000, 46, 1338-1344. [CrossRef] [PubMed]

15. Giannitsis, E.; Kurz, K.; Hallermayer, K.; Jarausch, J.; Jaffe, A.S.; Katus, H.A. Analytical Validation of a High-Sensitivity Cardiac Troponin T Assay. Clin. Chem. 2010, 56, 254-261. [CrossRef] [PubMed]

16. Koerbin, G.; Tate, J.R.; Hickman, P.E. Analytical characteristics of the Roche highly sensitive troponin T assay and its application to a cardio-healthy population. Ann. Clin. Biochem. 2010, 47, 524-528. [CrossRef]

17. Brown, L.D.; Cai, T.T.; DasGupta, A. Interval Estimation for a Binomial Proportion Author(s): Lawrence, D. Brown, T. Tony Cai, Anirban DasGupta Published by: Institute of Mathematical Statistics Stable. Stat. Sci. 2001, 16, 101-117. Available online: http:/ / www.jstor.org/stable/2676784 (accessed on 16 June 2020).

18. Janssen, K.; Moons, K.; Kalkman, C.; Grobbee, D.; Vergouwe, Y. Updating methods improved the performance of a clinical prediction model in new patients. J. Clin. Epidemiol. 2008, 61, 76-86. [CrossRef]

19. Steyerberg, E.W.; Vergouwe, Y. Statistical tutorials towards better clinical prediction models: Seven steps for development and an ABCD for validation. Eur. Heart J. 2014, 35, 1925-1931. [CrossRef] [PubMed]

20. Harrell, F.E.; Lee, K.L.; Califf, R.M.; Pryor, D.B.; Rosati, R.A. Regression modelling strategies for improved prognostic prediction. Stat. Med. 1984, 3, 143-152. [CrossRef]

21. Moons, K.G.; Kengne, A.P.; Grobbee, D.E.; Royston, P.; Vergouwe, Y.; Altman, D.G.; Woodward, M. Risk prediction models: II. External validation, model updating, and impact assessment. Heart 2012, 98, 691-698. [CrossRef] [PubMed]

22. Steyerberg, E.W.; Vickers, A.J.; Cook, N.R.; Gerds, T.; Gonen, M.; Obuchowski, N.; Pencina, M.J.; Kattan, M.W. Assessing the performance of prediction models: A framework for traditional and novel measures. Epidemiology 2010, 21, 128-138. [CrossRef]

23. Alba, A.C.; Agoritsas, T.; Walsh, M.; Hanna, S.; Iorio, A.; Devereaux, P.J.; McGinn, T.; Guyatt, G. Discrimination and calibration of clinical prediction models: Users' guides to the medical literature. JAMA-J. Am. Med. Assoc. 2017, 318, 1377-1384. [CrossRef]

24. Vickers, A.J.; Elkin, E.B. Decision Curve Analysis: A Novel Method for Evaluating Prediction Models. Med Decis. Mak. 2006, 26, 565-574. [CrossRef] [PubMed] 
25. Delong, E.R.; Delong, D.M.; Clarke-Pearson, D.L. Comparing the Areas under Two or More Correlated Receiver Operating Characteristic Curves: A Nonparametric Approach. Biometrics 1988, 44, 837. [CrossRef]

26. Wilson, E.B. Probable Inference, the Law of Succession, and Statistical Inference. J. Am. Stat. Assoc. 1927, 22, 209-212. [CrossRef]

27. Vergouwe, Y.; Steyerberg, E.W.; Eijkemans, M.J.; Habbema, J.D.F. Substantial effective sample sizes were required for external validation studies of predictive logistic regression models. J. Clin. Epidemiol. 2005, 58, 475-483. [CrossRef] [PubMed] 